\title{
Criminologie
}

\section{Les zones urbaines criminelles}

\section{Maurice Cusson}

Volume 22, numéro 2, 1989

Le milieu criminel

URI : https://id.erudit.org/iderudit/017283ar

DOI : https://doi.org/10.7202/017283ar

Aller au sommaire du numéro

Éditeur(s)

Les Presses de l'Université de Montréal

ISSN

0316-0041 (imprimé)

1492-1367 (numérique)

Découvrir la revue

\section{Citer cet article}

Cusson, M. (1989). Les zones urbaines criminelles. Criminologie, 22(2), 95-105. https://doi.org/10.7202/017283ar

\section{Résumé de l'article}

The study of criminal areas has been a longstanding tradition in sociology and criminology. It had its hour of glory during the publication of the works of Shaw and McKay of the University of Chicago. In this article - which is an account of the studies on the concentration of criminals in urban areas - we show that, since the XIXth century, large metropolises have relatively stable sectors where the social control is weak and opportunities to commit crimes are numerous. Networks of juvenile delinquents and adult criminals develop in these areas, fostering the transmission of criminal solutions. The article contains a description of the process that leads to the emergence of a crime zone. It ends with a critique on ecological studies and by an appeal for the study of conflicts within these criminal networks.
Ce document est protégé par la loi sur le droit d'auteur. L'utilisation des services d'Érudit (y compris la reproduction) est assujettie à sa politique d'utilisation que vous pouvez consulter en ligne.

https://apropos.erudit.org/fr/usagers/politique-dutilisation/ 
The study of criminal areas has been a longstanding tradition in sociology and criminology. It had its hour of glory during the publication of the works of Shaw and McKay of the University of Chicago. In this article - which is an account of the studies on the concentration of criminals in urban areas - we show that, since the $X I X^{\text {th }}$ century, large metropolises have relatively stable sectors where the social control is weak and opportunities to commit crimes are numerous. Networks of juvenile delinquents and adult criminals develop in these areas, fostering the transmission of criminal solutions. The article contains a description of the process that leads to the emergence of a crime zone. It ends with a critique on ecological studies and by an appeal for the study of conflicts within these criminal networks.

La sociologie criminelle telle qu'elle a été développée par l'École de Chicago posait le problème des interactions criminogènes en termes territoriaux. C'est parce que les délinquants vivaient concentrés dans les quartiers du centre de la ville qu'ils se transmettaient des modèles criminels d'une génération à l'autre. Aujourd'hui, les moyens de transport font reculer les contraintes de la proximité physique sans les supprimer pour autant. Cette tradition de recherche est loin d'être éteinte : le filon continue d'être exploité, comme en témoigne la publication de Communities and Crime (Reiss et Tonry eds, 1986). Elle reste une excellente introduction à l'analyse des interactions entre criminels.

Cet article est un bilan des travaux sur la concentration des délinquants dans le territoire urbain. Quel savoir s'en dégage ? Quelles sont les limites de cette approche ? Comment les dépasser?

C'est en 1929 que fut publié Delinquency Areas de Shaw. Plusieurs années plus tard, en 1942, Shaw s'associe avec McKay pour publier Juvenile Delinquency and Urban Areas qui allait devenir un classique. Pour l'essentiel, la méthode de ces deux sociologues consiste à situer sur la carte de Chicago les jeunes délinquants selon leur lieu de résidence. Ils constatent ainsi que la délinquance juvénile est très inégalement répartie sur le territoire urbain. Par exemple, pour la série de 1927 à 1933, les taux de délinquance par 100 garçons de 10 à 16 ans varient de 0,5 pour le secteur le plus bas à 18,9 pour le plus haut (p. 53). Les zones

* Professeur, Ecole de criminologie, Université de Montréal, case postale 6128, succursale A, Montréal, Québec, H3C 3J7. 
de la ville où la délinquance juvénile se concentre sont à l'intérieur des zones industrielles et commerciales ou adjacentes à elles. La concentration de jeunes délinquants est très élevée au centre de la ville et elle diminue progressivement au fur et à mesure qu'on se dirige vers sa périphérie.

Pour rendre compte de leurs observations, Shaw et McKay proposent une théorie dite de la transmission culturelle. L'intégration sociale des communautés du centre de la ville est minée par les activités commerciales et industrielles qui s'y implantent ainsi que par l'afflux des divers groupes qui s'y installent. Il y émerge alors un système de valeurs qui entre en compétition avec les valeurs conventionnelles qui, elles, dominent sans partage dans les quartiers résidentiels périphériques. Au sein des quartiers où se concentrent les jeunes délinquants, le crime s'affiche sans vergogne; les gangs font la loi ; les «rackets» fleurissent; les receleurs sont connus; les criminels adultes s'affichent. Il devient alors difficile aux parents, aux maîtres, aux membres du clergé de défendre auprès de la jeunesse des valeurs qui sont constamment concurrencées par des valeurs antinomiques. C'est ainsi que, génération après génération, des modèles culturels anti-socjaux sont transmis aux adolescents des zones délinquantes.

S'inspirant de Shaw et de McKay, Sutherland (1939) devait faire avancer d'un pas de plus la question en proposant sa théorie de l'association différentielle. Le criminel a subi un apprentissage au cours duquel des camarades avec qui il entretient des rapports personnels l'initient à des techniques criminelles et le nourrissent d'attitudes et de justifications favorables au crime. On devient donc criminel parce que l'on a été exposé à des systèmes d'interprétation qui présentent la transgression des lois sous un jour favorable plus souvent, plus longtemps, plus précocement et plus intensément qu'à des systèmes d'interprétation qui condamnent le crime.

La théorie à laquelle on aboutit dans celle tradition est donc un effort pour expliquer les propensions criminelles (et non l'acte) en termes d'apprentissage d'éléments sous-culturels.

Chicago n'est évidemment pas la seule ville où on observe des concentrations de criminels dans l'un ou l'autre quartier. La plupart des grandes villes anglaises et américaines du $19^{\mathrm{e}}$ et du $20^{e}$ siècles, sur lesquelles des recherches ont été menées, comportaient un ou plusieurs secteurs où se concentraient jeunes délinquants et criminels adultes (Brantingham et Brantingham 1984, p. 298 à 326). Les quartiers criminels de Londres, appelés «Rookeries», étaient particulièrement célèbres 
aux $18^{\mathrm{e}}$ et $19^{\mathrm{e}}$ siècles. C'étaient des quartiers des rues obscures, avec des tavernes remplies d'individus louches, des hôtels mal famés et des bordels. C'était là que se réfugiaient les clochards, les prostituées, les voleurs, les receleurs et les assassins (Chesney, 1972; Tobias, 1967). Ils servaient de sanctuaire aux criminels parce qu'il était difficile de s'y retrouver, qu'il était dangereux pour les honnêtes gens d'y circuler et que les forces de l'ordre n'osaient y pénétrer.

Les zones criminelles présentent trois caractéristiques : elles sont relativement stables; le contrôle social y est faible et les opportunités criminelles sont accessibles. La stabilité des zones criminelles est frappante. À Londres, Whitechapel et Goldenlane, deux quartiers considérés comme des «rookeries» au début du $19 \mathrm{e}$ siècle, fournissent, encore en 1960, des jeunes délinquants en surnombre (Brantingham et Brantingham 1984, p. 300). À Chicago, Shaw et McKay (1942) trouvent sensiblement la même distribution de jeunes délinquants entre 19001906 et 1924-1926. Dans la réédition de 1969 de leur ouvrage, ils présentent des cartes datées de 1958-1966 qui ont une configuration semblable aux précédentes. Or pendant toutes ces années, la composition ethnique des quartiers de Chicago ne cessait de se tranformer.

Les zones criminelles jouissent d'une immunité certaine vis-à-vis des agents de contrôle social. Les pressions sociales qui, normalement, s'exercent dans les familles, dans les écoles et dans les communautés locales n'y agissent que faiblement dans ces zones. Les forces de l'ordre éprouvent de la difficulté à s'y enraciner.

La criminalité doit s'alimenter à un réservoir d'opportunités. Aux États-Unis, les zones criminelles sont presque toujours situées au centre-ville, donc à proximité des commerces et des foules de citadins qui vont travailler ou faire des achats au coeur de la ville. Les ports et les quartiers d'amusement où se trouvent des discothèques, des bars et des bordels sont aussi des sites favorables à l'émergence d'une zone criminelle.

La dynamique criminogène de ces zones découle de l'action convergente de deux processus : l'émergence de réseaux criminels et l'affaiblissement des contrôles sociaux.

Shaw et McKay (1942 p. 174) avaient mis en relief le fait que, dans les zones délinquantes, la densité de jeunes délinquants est telle qu'un garçon qui y vit entre presque nécessairement en contact avec des délinquants. Ainsi peuvent se constituer des réseaux qui mettent en rapport les délinquants les uns avec les autres. La concentration de crimi- 
nels dans le territoire urbain produit un effet de masse critique : quand un nombre suffisamment élevé d'individus portés au crime sont mis en présence les uns des autres, il se produit une accélération de la dynamique criminelle de chacun.

Les caractéristiques des quartiers urbains à forte criminalité sont assez bien connues grâce à de nombreuses recherches. Le tableau tient en douze caractéristiques.

1. Dans ces quartiers, les taux de familles monoparentales, de divorces et de naissances illégitimes sont particulièrement élevés;

2. Les pourcentages de locataires sont supérieurs à ceux qu'on trouve dans les autres secteurs de la ville étudiée;

3. Les ménages formés d'un individu vivant seul sont en surnombre;

4. Les pourcentages d'immeubles à logements multiples, par exemple des HLM, y sont particulièrement élevés;

5. L'alcoolisme y apparaît comme un sérieux problème;

6. Il s'y commet plus de suicides que dans les autres secteurs de la ville;

7. Les immigrants ainsi que les minorités sociales ou ethniques ont tendance à se concentrer dans ces secteurs;

8. La mobilité résidentielle y est relativement forte;

9. Les logements et les équipements collectifs sont mal entretenus et quelquefois sont dans un état de délabrement avancé ;

10. La population est à la baisse;

11. La dépendance des prestations de la sécurité sociale y est plus fréquente qu'ailleurs;

12. Les inégalités économiques y sont plus accentuées que dans les autres quartiers (Voir Shaw et McKay 1942; Sampson, 1983, 1987; Brantingham et Brantingham 1984; Reiss et Tonry 1986 eds).

Familles éclatées, habitat délabré, individus désespérés, populations disparates, il est clair que, dans de tels quartiers, les conditions nécessaires au développement d'un sentiment communautaire sont totalement absentes. Nous sommes en présence de gens écrasés par la dure- 
té de la vie transitant dans un quartier où ils n'ont pas pris racine. Ils sont entourés d'étrangers et ils vivent isolés et repliés sur eux-mêmes. Dans ce climat marqué par l'isolement, le déracinement et l'impuissance, les contrôles sociaux seront nécessairement très faibles.

Aux États-Unis, le processus qui conduit à l'émergence d'une zone criminelle est maintenant assez bien connu. La caractéristique essentielle de cette évolution est que le centre des villes se vide progressivement de ses familles stables et de ses citadins de classe moyenne ou supérieure qui sont remplacés par des familles fragiles, par des gens en difficulté et par des déviants. Ce processus peut être découpé en six étapes ${ }^{l}$.

1. Les premiers signes de détérioration. Il apparaît un jour que l'habitat d'un secteur central de la ville commence à se dégrader: les maisons vieillissent et on néglige de faire les réparations nécessaires; les parcs, les écoles et les autres lieux publics sont moins bien tenus et se délabrent peu à peu. Il peut arriver qu'un événement singulier altère brusquement la qualité du quartier, par exemple, la construction d'une autoroute qui traverse le secteur, forçant la démolition de nombreuses résidences et défigurant le quartier. Un mouvement de désaffection se fait alors sentir : on investit de moins en moins dans la construction et la rénovation des résidences ainsi que dans le renouvellement des équipements publics. On a de plus en plus le sentiment que la qualité de la vie du quartier n'est plus ce qu'elle était.

2. Les changements sélectifs de population. Peu à peu, les gens qui ont les moyens de s'installer dans un environnement plus agréable déménagent. De nombreuses familles de classe moyenne abandonnent le quartier, ce qui provoque une baisse des prix des logements. Attirée par les loyers modiques, une nouvelle population commence à s'installer dans le quartier : célibataires, familles mono-parentales, vieillards, étrangers, marginaux. Ceci accentue le mouvement qui pousse les families stables à fuir le secteur. Aux États-Unis, de tels changements résidentiels sont quelquefois très rapides à cause de la forte mobilité géographique des Américains qui sont peu attachés à leur communauté natale et assez attirés par les banlieues. C'est pourquoi, aux États-Unis, la dégradation d'un quartier peut déclencher assez rapidement un exode massif parmi les classes moyennes.

1. Ces six étapes sont une manière de décrire un processus évolutif. Il ne faut pas y voir une séquence rigoureuse où chaque étape succéderait nécessairement à l'autre. Les meilleures analyses de ce phénomène se trouvent dans Skogan (1986) et Reiss (1986). 
3. L'affaiblissement des contrôles sociaux. La capacité de la communauté locale de faire face à ses problèmes s'étiole, car elle perd peu à peu ses résidents qui avaient les moyens de se mobiliser. On cesse de faire pression sur les élus locaux et sur les autorités régionales pour que les équipements publics soient mieux entretenus et pour que la qualité des services publics soit rehaussée. On laisse s'installer des trafics et des commerces qui donnent une mauvaise réputation au secteur : prostitution, bars, «sex shop»... Des adolescents laissés à eux-mêmes se regroupent, commettent de menus larcins, se livrent au vandalisme et intimident les passants.

4. La peur, la méfiance et l'impuissance. S'instaure alors dans les rues un climat de peur et de méfiance engendré par l'apparence physique du quartier et par la conduite des gens dans les lieux publics: saleté des rues, maisons abandonnées aux vandales, graffiti, alcooliques qui titubent dans la rue, prostituées qui racolent des clients, groupes de jeunes gens qui insultent les filles et intimident les passants. La délinquance a un rôle important à jouer ici. Il a été démontré que la peur du crime n'est pas sans rapport avec les taux réels de criminalité. Les citoyens qui disent avoir peur de se promener le soir dans les rues de leur quartier se concentrent précisément dans les secteurs de la ville ayant les taux de criminalité les plus élevés (Skogan et Maxfield, 1981). Il y a donc une corrélation entre les taux de crimes et la peur du crime. La peur et la méfiance sont particulièrement prononcées quand les citadins pensent que ce sont des habitants de leur propre quartier qui sont responsables de la délinquance locale et non des individus venus d'ailleurs.

Le sentiment d'insécurité accélère le mouvement de fuite du quartier. Ceux qui n'ont pas les moyens de déménager évitent de sortir le soir. Quand ils doivent sortir, ils adoptent les trajets qui leur paraissent les plus sûrs et ils évitent de parler aux gens qu'ils ne connaissent pas. Ainsi les rues se vident-elles, laissant le champ libre aux voyous. Les quelques passants qui s'aventurent dans les rues désertées deviennent vulnérables, ne pouvant obtenir de l'aide si on fait mine de les attaquer.

5. La spirale du crime. L'érosion des contrôles sociaux fait monter d'un cran la criminalité locale. Elle s'organise. Le quartier attire une population de plus en plus marginale : alcooliques, voyous, vagabonds, prostitué(e)s, bandits, trafiquants de drogues. Les criminels recherchés par la police viennent y trouver refuge. La densité de délinquants et de marginaux permet le développement d'une véritable organisation sociale du crime. Des réseaux se forment; des marchés de substances illégales et de biens volés fleurissent. Les modèles criminels exercent une 
attraction que ne sauraient contrebalancer les quelques éléments honnêtes qui persistent à rester dans la zone.

6. L'effondrement. Skogan (1986) a particulièrement bien décrit le terme du processus. Le crime et le désordre deviennent les facteurs dominants dans le quartier. La peur est devenue omniprésente. Pour vivre dans un quartier qui a atteint ce point (par exemple le «South Bronx» de New-York), il faut une telle tolérance au risque que la population chute rapidement. Ne restent plus que les clochards, les «junkies» et les bandits. Des édifices abandonnés sont incendiés. D'autres sont utilisés pour la distribution et la consommation de drogues. Et alors la criminalité se met à baisser tout simplement parce que les cibles deviennent de plus en plus rares; il n'y a plus grand chose à voler ni grand monde à agresser.

Aux États-Unis, l'évolution qui vient d'être décrite a été un facteur déterminant dans le gonflement des banlieues et dans la détérioration concomitante des centres villes. Dans quelques cas extrêmes, le centre de certaines grandes villes américaines devient la nuit un véritable «no man's land». Skogan (1977, cité par Gurr, 1981) a d'ailleurs montré que les villes dont les banlieues se sont le plus développées entre 1948 et 1970 ont connu la plus forte croissance de la criminalité. Nous sommes ici en présence d'un effet pervers tel que le conçoit Boudon (1977). Les citoyens, craignant le crime dans la ville, fuient vers la périphérie pour y trouver la sécurité mais, ce faisant, ils abandonnent un secteur stratégique de la cité - son centre - aux voyous et aux criminels qui peuvent alors à loisir piller et agresser tout ce qui reste à leur portée. À l'échelle individuelle, la décision de se réfugier en banlieue paraît rationnelle mais, à l'échelle de la société, elle contribue à la croissance de la criminalité et de l'insécurité.

Dans ce qui suit, j'indiquerai d'abord les limites des recherches qui viennent d'être présentées. Je soulignerai ensuite l'importance de l'analyse des conflits pour accéder à une meilleure connaissance des interactions entre les criminels. Mes remarques porteront sur quatre points.

1: Felson (1983) a fait observer que les premières études écologiques du crime avaient été menées à une époque où l'automobile était un luxe et où la plupart des activités de la vie courante se déroulaient au sein d'un même quartier. Avec la croissance énorme du parc automobi- 
le et celui des autres moyens de transport, les activités quotidiennes des citadins se sont éparpillées sur un espace considérable; les banlieues se sont développées et les distances entre le lieu de travail, de la résidence et des loisirs n'ont cessé de grandir. Les quartiers de nos villes ressemblent de moins en moins aux villages urbains qu'ils étaient au début du siècle. Ceci ne veut pas dire que les contraintes spatiotemporelles cessent de s'exercer. Ce n'est pas par téléphone que s'établissent les rapports d'amitié et de collaboration qui lient les délinquants entre eux. Du moins le téléphone ne suffit pas. Le contact physique reste nécessaire, mais il obéit à d'autres lois que celles de la simple proximité résidentielle. Où les membres du milieu criminel entrent-ils en contact les uns avec les autres ? Dans les prisons? Dans certains bars ? Dans certains restaurants ? L'étude des lieux où se rencontrent les criminels reste à faire, sans doute parce qu'elle est difficile.

2. La sociologie criminelle traditionnelle fut excessivement culturaliste. Le criminel était conçu comme un conformiste qui a trop bien intériorisé les valeurs de sa sous-culture. Mais l'explication était à la limite de la tautologie : on devient criminel à la suite d'un apprentissage de normes et de valeurs qui ne sont pas mesurées autrement que par le comportement que l'on veut expliquer. Il me semble que Hirschi et Gottfredson (1988, p. 22) ont raison de penser que la fréquentation de pairs délinquants a peu d'influence sur la propension au crime, elle agit plutôt par le biais des situations pré-criminelles. Les activités d'un groupe de délinquants (par exemple, flâner dans la rue à $2 \mathrm{~h}$ du matin) mettent en présence de situations favorables au passage à l'acte. L'influence de pairs délinquants apparaît donc beaucoup plus instrumentale que normative. Celui qui est intégré dans un réseau criminel se trouve facilement des complices: il a de bons «tuyaux», il sait où se procurer des armes, il a un fournisseur de drogue... Tout ceci augmente la probabilité que son activité délinquante produise les résultats escomptés. Bref, les criminels se transmettent, non pas des idéaux, mais des solutions.

3. La criminologie est sillonnée de fausses pistes : le crime organisé, les conflits de culture, les criminels de carrière... Il est deux de ces pistes qui partent dans des directions opposées, mais qui ont en commun de mener nulle part. La première se donne pour objet un individu délinquant parfaitement isolé, sans rapport avec le monde. La seconde part de l'a priori fort discutable selon lequel les criminels se regroupent dans des structures parfaitement organisées, hiérarchisées et intégrées. L'analyse doit se garder de se fixer soit sur des individus isolés, soit sur une entité supra-individuelle dont la réalité n'est point assurée. C'est 
sur les actions et les réactions des délinquants que l'on devrait faire porter l'attention. Aussi P. Tremblay a-t-il raison de définir le milieu criminel par «l'ensemble des interactions directes ou indirectes entre des individus ayant choisi de participer de façon relativement régulière à un ensemble variable d'activités criminelles». Il s'agit donc d'étudier l'activité de relation qui gravite autour de la délinquance instrumentale récurrente. La recherche portant sur un objet ainsi défini ne peut tout simplement plus se confiner à l'empirisme pur qui continue de sévir en criminologie. Un univers relationnel s'appréhende avec des concepts, sinon il nous glisse entre les doigts. Le concept de marché que nous empruntons des économistes est un instrument pour analyser une activité relationnelle mais il n'est évidemment pas le seul. La notion de conflit est aussi fort utile.

4. Contrairement à l'image idyllique que peignait Sutherland, (1937) les milieux criminels sont constamment traversés de conflits violents. Sur ce point, les premiers victimologues n'ont pas manqué de perspicacité. Von Hentig (1948) et Ellenberger (1954) faisaient observer que les criminels sont souvent victimes et inversement. Le criminel, écrivait Ellenberger, est "prédisposé au rôle de victime» (p. 104). C'est un fait que la pègre génère une criminalité qui frappe ses propres membres. Récemment, l'américain Singer (1981) rapportait une étroite corrélation entre le fait d'avoir été l'auteur de coups et blessures et celui d'être victime d'un coup de feu ou d'un coup à l'arme blanche. Les victimes de violence sont deux fois et demie plus souvent des délinquants violents que les non-victimes. Dans les données de cet auteur, l'expérience de la victimisation pendant l'adolescence est un très bon prédicteur de la délinquance adulte violente : $64 \%$ des individus qui, à l'adolescence, avaient été victimisés, commettent, devenus adultes, des coups et blessures, contre $22 \%$ chez les adolescents nonvictimes (p. 782). En Angleterre, Gottfredson (1984), analysant les données du «British Crime Survey», fait de semblables constatations. La probabilité de victimisation des délinquants est sept fois plus élevée que celle des non-délinquants. Les criminels se victimisent les uns les autres de plusieurs manières. Les voies de fait au cours de bagarres sont probablement les infractions les plus fréquentes. Dans de telles affaires, la différence entre le criminel et sa victime ne saurait être bien grande. Souvent, "c'est le hasard qui décidera si le sujet sera criminel ou victime» (Ellenberger 1954, p. 105). Les vols (de drogue, de biens volés, etc...) sont d'autant plus fréquents au sein de la pègre que la victime est généralement en fort mauvaise posture pour dénoncer son voleur à la police... Au pinacle de cette criminalité endogène au milieu, trône le règlement de comptes tel que l'entend Cordeau (1989), c'est-à-dire le 
meurtre qui s'inscrit dans un conflit résultant de la participation du meurtrier et de sa victime à une activité criminelle.

Comment expliquer que les criminels soient si souvent victimes de leur propre médecine ? La proximité est un premier facteur. En effet les délinquants vivent souvent dans les mêmes lieux, aux mêmes heures et ils ne vont pas chercher très loin leurs victimes. Leurs pairs antisociaux sont donc des cibles probables. On aurait pu s'attendre à ce que, par solidarité, les membres du milieu s'interdisent mutuellement le recours à la ruse et à la force. L'instauration d'un régime de respect réciproque eût été avantageux pour tous. Mais Hobbes nous avait appris qu'en l'absence d'une autorité supérieure qui garantit les engagements des uns vis-à-vis des autres, la méfiance et l'hostilité prévalent. Contrairement à ce que pourrait laisser croire la mythologie du crime organisé, il n'existe pas, dans le milieu, de pouvoir suprême qui puisse mettre un terme à la guerre de tous contre tous. Dans ces conditions, les criminels apprennent avec le temps à maintenir une bonne distance entre eux et leurs congénères pour assurer leur sécurité. On comprend alors pourquoi, en vieillissant, le délinquant a tendance à agir plus souvent seul. Alors que, dans l'état civil, plus on est proche de ses semblables, plus on est en sécurité, dans l'état de nature, plus on est proche de ses semblables, plus on s'expose. Il est inévitable que la violence serve à résoudre les conflits parmi des gens qui se côtoient, qui se connaissent et qui ne jouissent pas de la protection de l'État. «La Bible nous montre Caïn marqué d'un signe sur le front pour l'empêcher d'être tué par ses semblables. C'est le criminel professionnel qui vit le plus dangereusement puisqu'il se tient en dehors de la protection de la Société» (Ellenberger 1954, p. 104). Exclus de la justice, les criminels ne disposent pas des moyens judiciaires pour régler leurs disputes. Ils sont alors contraints de prendre la justice entre leurs mains sans pour autant disposer des mécanismes institutionnels freinant la vengeance que connaissaient les sociétés vindicatives d'autrefois (Cusson 1987, chap. IV). Ce qu'on appelle une guerre de gang ressemble fort à une série de vengeances en chaîne.

\section{RÉFÉRENCES}

BOUDON, R. (1977), Effets pervers et ordre social, Paris: Presses universitaires de France.

BRANTINGHAM, P. BRANTINGHAM, P. (1984), Patterns in Crime, New York: MacMillan.

CHESNEY, K. (1970), The Victorian Underworld, Londres : Temple Smith (Traduction française : Les Bas-fonds victoriens, Laffont, 1981). 
CORDEAU, G. (1989), «Les mécanismes de la dissuasion endogène», Revue Canadienne de criminologie, 31, 3, 253-280.

CUSSON, M. (1987), Pourquoi punir ? Paris: Dalloz.

ELLENGERGER, H. (1954), «Relations psychologiques entre le criminel et la victime», Revue internationale de criminologie et de police technique, vol. XIII $\mathrm{n}^{\circ} \mathrm{I}, 103-121$.

FELSON, M. (1983), «Ecology of Crime» in Encyclopedia of Crime and Justice, vol. II, New York : The Free Press.

GOTTFREDSON, M. (1984), Victims of Crime : The Dimensions of Risk. London : H.M. Stationery Office.

GURR, T. (1981), «Historical Trends in Violent Crimes: A Critical Review of the Evidence", in Tonry, M. and Morris, N. : Crime and Justice : An annual Review of Research, vol. 3, Chicago : University of Chicago Press, 295-353.

HIRSCHI, T., GOTTFREDSON, M. (1988), «Towards a general theory of crime», in BUIKHUISEN, W., MEDNICK, S.A. (eds), Explaining Criminal Behaviour, Leiden : E.J. Brill.

REISS, A. (1986), "Why are Communities Important in Understanding Crime?» in REISS, A.; TONRY, M. (eds), Communities and Crime, Chicago: University of Chicago Press, 1.33.

REISS, A., TONRY, M. (eds) (1986), Communities and Crime, Chicago : University of Chicago Press.

SAMPSON, R.J. (1983), «Structural Density and Criminal Victimization», Criminology, vol. 21, 276-293.

SAMPSON, R.J. (1987), «Communities and Crime» in GOTTFREDSON, M.R. and HIRSCHI, T., Positive Criminology, Beverly Hills, Calif. : Sage, 91-114.

SHAW, C.R. (1929), Delinquency Areas, Chicago : University of Chicago Press.

SHAW, C.R., McKAY, H.D. (1942), Juvenile Delinquency and Urban Areas, Chicago : University of Chicago Press.

SINGER, S.I. (1981), «Homogeneous Victims-Offenders Population: A Review and Some Research Implications", Journal of Criminal Law and Criminology, 72 : 779-88.

SKOGAN, W.G. (1986), "Fear of Crime and Neighborhood Change», in REISS, A., TONRY, M. (eds), Communities and Crime, Chicago : University of Chicago Press, 203-229.

SUTHERLAND, E.H. (1937), The Professional Thief, Chicago : University of Chicago Press, (Traduction française : Spes, Paris, 1963).

SUTHERLAND, E.H. (1939), Principles of criminology, Philadelphia : Lippincott.

TOBIAS, J.J. (1967), Crime and Industrial Society in the Nineteenth Century, London : Batsford.

VON HENTIG, H. (1948), The Criminal and His Victim, New Haven : Yale University Press. 